\title{
Common polymorphisms in SOCS3 are not associated with body weight, insulin sensitivity or lipid profile in normal female twins
}

Received: 8 June 2005 / Accepted: 19 October 2005 / Published online: 10 January 2006

(C) Springer-Verlag 2006

\begin{abstract}
Aims/hypothesis: Inhibition of signal transduction by suppressor of cytokine signalling-3 (SOCS-3) potentially influences resistance to insulin and leptin. The aim of this study was to test the association between three single-nucleotide polymorphisms (SNPs) representative of common linkage disequilibrium clusters in SOCS3 (rs4969169, rs12953258 and rs8064821) and obesity measures, insulin sensitivity measures and serum lipids in the general population. Methods: The three SNPs, which had rare allele frequencies $>0.06$, were genotyped in 2,777 female twins of European extraction (mean age $47.4 \pm 12.5$ years) from the St Thomas' UK Adult Twin Registry (Twins UK). Results: Minor allele frequencies were as follows: rs4969169 $=0.067$, rs12953258 $=0.097$ and rs8064821 $=0.101$. Individual SOCS3 SNPs were not associated with general or central obesity, or with two indices of insulin sensitivity (homeostasis model assessment and insulin sensitivity measure). Conclusions/
\end{abstract}

Electronic Supplementary Material Supplementary material is available for this article at http://dx.doi.org/10.1007/s00125-0050093-3.

Y. Jamshidi · N. D. Carter · S. D. O’Dell

Department of Clinical Developmental Sciences

St George's, University of London,

London, UK

Y. Jamshidi · S. D. O’Dell ( $ه)$

Nutrition Food and Health Research Centre,

King's College London,

Franklin-Wilkins Building,

150 Stamford Street,

SE1 9NH London, UK

e-mail: sandra.o'dell@kcl.ac.uk

Tel.: +44-207-8483177

Fax: +44-207-8484185

H. Snieder - X. Wang

Department of Pediatrics, Georgia Prevention Institute,

Medical College of Georgia,

Augusta GA, USA

H. Snieder · T. D. Spector

Twin Research and Genetic Epidemiology Unit,

St Thomas' Hospital,

London, UK interpretation: The results do not indicate that any of the three SNPs studied are associated with obesity, insulin measures or lipid measures.

Keywords Insulin resistance · Insulin sensitivity · Obesity $\cdot$ Weight regulation

Abbreviations apoAI: apolipoprotein AI - D': pairwise LD statistic · ESM: electronic supplementary material · HOMA: homeostasis model assessment - LD: linkage disequilibrium - sib-TDT: sibling transmissiondisequilibrium test - SiM: insulin sensitivity measure . SNP: single-nucleotide polymorphism - SOCS-3: suppressor of cytokine signalling-3 - VD: distribution volume

\section{Introduction}

Suppressor of cytokine signalling-3 (SOCS-3) blocks the access of signal transducers and activators of transcription to cytokine receptor binding sites [1] and has been implicated in leptin/insulin resistance [for review see 2, 3]. A recent case-control study reported that homozygosity for the A allele of the C-920A (rs12953258) promoter polymorphism of SOCS3 was associated with increased whole-body insulin sensitivity in young Danish subjects [4]. In an attempt to substantiate this observation and to look for an association between obesity and this gene, we validated two further single-nucleotide polymorphisms (SNPs) in this gene and analysed the association between the three SNPs and obesity or insulin resistance measurements in 2,777 women from the St Thomas' UK Adult Twin Registry.

\section{Subjects and methods}

Study design

The St Thomas' UK Adult Twin Registry (Twins UK) comprises unselected, mostly female, volunteers taken 
from the general population through national media campaigns in the UK [5]. Means and ranges of quantitative phenotypes in Twins UK were similar to those in an agematched sample from the general population [6] and the study cohort was selected on the basis of available leptin data. Informed consent was obtained from participants before they entered the cohort and the study was approved by the local research ethics committee. General characteristics of the study cohort are given in Table 1. A total of 261 non-fasting subjects and 28 patients with either type 1 or type 2 diabetes were excluded from the analysis of variables related to insulin sensitivity and 16 subjects using lipid-lowering agents were excluded from the analysis of lipid profiles.

\section{Zygosity, body composition and biochemical analyses}

Zygosity was determined by standardised questionnaire and confirmed by DNA fingerprinting. Body composition was measured by dual emission X-ray absorptiometry (Hologic QDR-2000, Vertec, Waltham, MA, USA). Serum leptin concentration was determined after an overnight fast using a radioimmunoassay (Linco Research, St Louis, MO, USA). Levels of HDL cholesterol and triglycerides were

Table 1 General characteristics of subjects

\begin{tabular}{|c|c|c|}
\hline Variable & Number & Mean \pm SD \\
\hline Age (years) & $2,777^{\mathrm{a}}$ & $47.4 \pm 12.5$ \\
\hline Postmenopausal (\%) & 2,454 & 47.7 \\
\hline \multicolumn{3}{|l|}{ Obesity-related variables: } \\
\hline Leptin (ng/ml) & 2,777 & $16.5 \pm 12.0$ \\
\hline BMI $\left(\mathrm{kg} / \mathrm{m}^{2}\right)$ & 2,761 & $24.8 \pm 4.4$ \\
\hline Weight (kg) & 2,762 & $65.3 \pm 11.8$ \\
\hline Waist (cm) & 2,710 & $78.4 \pm 10.2$ \\
\hline Total fat $(\mathrm{kg})$ & 2,721 & $23.4 \pm 8.8$ \\
\hline Total fat $(\%)$ & 2,680 & $35.6 \pm 8.0$ \\
\hline Central fat $(\mathrm{kg})$ & 2,698 & $1.33 \pm 0.73$ \\
\hline Central fat $(\%)$ & 2,698 & $31.2 \pm 11.5$ \\
\hline \multicolumn{3}{|l|}{ Insulin sensitivity ${ }^{\mathrm{b}}$ : } \\
\hline Fasting glucose (mmol/l) & 2,322 & $4.49 \pm 0.56$ \\
\hline Fasting insulin (pmol/1) & 1,994 & $6.90 \pm 5.40$ \\
\hline HOMA & 1,785 & $1.34 \pm 1.10$ \\
\hline $2 \mathrm{~h}$ glucose $(\mathrm{mmol} / \mathrm{l})$ & 733 & $5.18 \pm 1.09$ \\
\hline $2 \mathrm{~h}$ insulin $(\mathrm{pmol} / \mathrm{l})$ & 733 & $33.4 \pm 23.2$ \\
\hline $\operatorname{SiM}\left(\times 10^{11} 1 \mathrm{pmol}^{-1} \mathrm{mmol}^{-1}\right)$ & 733 & $89.2 \pm 69.0$ \\
\hline \multicolumn{3}{|l|}{ Lipid profile ${ }^{c}$ : } \\
\hline Triglyceride (mmol/l) & 2,488 & $1.27 \pm 0.80$ \\
\hline Total cholesterol (mmol/l) & 2,600 & $5.57 \pm 1.25$ \\
\hline HDL cholesterol (mmol/l) & 2,609 & $1.55 \pm 0.39$ \\
\hline ApoA1 (g/l) & 2,436 & $1.70 \pm 0.34$ \\
\hline ApoB (g/l) & 2,454 & $1.17 \pm 0.36$ \\
\hline
\end{tabular}

${ }^{a}$ Number of subjects (846 monozygous twins, 1,931 dizygous twins) with leptin data and genotype data on at least one SNP; ${ }^{b}$ nonfasting subjects $(\leq 8 \mathrm{~h})$, patients with type 1 or type 2 diabetes and patients on any glucose-lowering drugs were excluded; ${ }^{c}$ subjects using lipid-lowering agents were excluded measured using a Cobas Fara machine (Roche Diagnostics, Lewes, UK) [7]. Fasting insulin was measured by immunoassay (Abbott Laboratories Ltd., Maidenhead, UK) and glucose was measured on an Ektachem 700 multichannel analyser using an enzymatic colorimetric slide assay (Johnson and Johnson Clinical Diagnostic Systems, Amersham, UK). A sub-sample of approximately 750 subjects, representing unselected female twins from the general population, underwent an oral glucose tolerance test (OGTT) for which glucose and insulin levels were measured before and $2 \mathrm{~h}$ after a 75 -g oral glucose load [8].

Genotyping for SNP validation and haplotype determination

We tested the presence of nine validated SNPs from two public sources: the NCBI database (http://www.ncbi.nlm. nih.gov/ SNP) and http://innateimmunity.net/ (SNP6157). Validation in the Twins UK cohort was attempted by genotyping eight random unrelated subjects using PCR and restriction at natural sites or at sites forced by mismatched PCR primers. The relative positions of the eight tested SNPs with respect to the first coding base in exon 2 are shown in parentheses: rs4969170 (-5362); rs7502530 (-3569); rs8064821 (-2215); rs12953258 (-920); rs12059 (287); rs1061489 (372); rs2280148 (796); SNP6157 (1050); rs4969169 (1267). Three SNPs with minor allele frequencies $>0.06$ were genotyped in 94 unrelated twin subjects randomly drawn from the Twins cohort, for determination of allele frequency and pairwise linkage disequilibrium. Primers and PCR conditions for SNP validation are given in Electronic supplementary material (ESM) Table 1.

Genotyping in cohort

The three SNPs rs4969169, rs8064821 and rs12953258 were genotyped in the complete cohort by Pyrosequencing, (Biotage, Uppsala, Sweden). Genotyping accuracy was assessed by the inclusion of duplicates (pairs of monozygotic twins) in the arrays and negative controls (water blanks) were included on each plate. The genotyping success rates (with error rates in parentheses) were 94.2\% $(0 \%)$ for rs $4969169,92.8 \%(0 \%)$ for $\mathrm{rs} 12953258$, and 95.9\% (2.2\%) for rs 8064821 . Primers and PCR conditions for genotyping by Pyrosequencing are provided in ESM Table 2.

\section{Statistical analysis}

Pairwise linkage disequilibrium between the three SNPs was tested by calculating D'. Association analyses in the full cohort including both twin subjects from each pair were performed using Generalized Estimating Equations, a regression technique that takes the non-independency of the twin data into account and yields unbiased $p$ values [9]. 
Table 2 SOCS3 genotype counts and frequencies of alleles and haplotypes

\begin{tabular}{|c|c|c|c|c|c|}
\hline \multirow[t]{2}{*}{$\mathrm{SNP}^{\mathrm{a}}$} & \multicolumn{3}{|l|}{ Genotype $^{\mathrm{c}}$} & \multirow[t]{2}{*}{ MAF } & \multirow{2}{*}{$\begin{array}{l}\text { HWE } \\
(p \\
\text { value })\end{array}$} \\
\hline & 11 & 12 & 22 & & \\
\hline rs4969169 & 1927 & 283 & 8 & 0.067 & 0.48 \\
\hline rs12953258 & 1778 & 390 & 16 & 0.097 & 0.28 \\
\hline rs8064821 & 1819 & 419 & 19 & 0.101 & 0.34 \\
\hline Haplotype $^{\text {b }}$ & rs4969169 & rs12953258 & rs8064821 & Frequency & \\
\hline 1 & 1 & 1 & 1 & 0.797 & \\
\hline 2 & 1 & 1 & 2 & 0.098 & \\
\hline 3 & 2 & 2 & 1 & 0.057 & \\
\hline 4 & 1 & 2 & 1 & 0.036 & \\
\hline 5 & 2 & 1 & 1 & 0.009 & \\
\hline 6 & 1 & 2 & 2 & 0.002 & \\
\hline
\end{tabular}

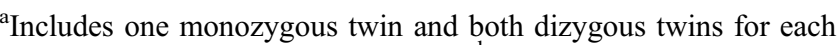
pair ( $n$ ranges from 2,184 to 2,257); ${ }^{\text {bincludes one monozygous }}$ twin and both dizygous twins for each pair with genotype data on at least two SNPs $(n=2,325) ;{ }^{c} 1=$ major allele, $2=$ minor allele. HWE, Hardy-Weinberg equilibrium; MAF, minor allele frequency
For individual SNP association analyses only a dominant model was tested because minor allele frequencies were low (6-11\%). Factor analysis was used to combine strongly correlated indices of obesity into two measures: one for general obesity (serum leptin, BMI, weight, total fat mass and percent total fat) and one for central obesity (waist, central fat mass and percentage central fat). We used two indices of insulin sensitivity: the homeostasis model assessment (HOMA), which is based on fasting data (HOMA=[fasting glucose $\times$ fasting insulin] / 22.5), and the insulin sensitivity measure (SiM) [10]. The calculation of SiM is based on both fasting and 2-h insulin and glucose data according to the following formula (in which $\mathrm{SiB}$ is the insulin sensitivity [fasting] at baseline; $\mathrm{SiH} 2$ is the insulin sensitivity $2 \mathrm{~h}$ after OGTT and VD is the distribution volume $): \mathrm{SiM}=([0.137 \times \mathrm{SiB}]+\mathrm{SiH} 2) / 2$, where $\mathrm{SiB}=108 /$ (fasting insulin $\times$ fasting glucose $\times \mathrm{VD}), \mathrm{SiH} 2=108 /(2-\mathrm{h}$ insulin $\times 2$-h glucose $\times \mathrm{VD}$ ) and $\mathrm{VD}=150 \mathrm{ml} / \mathrm{kg} \times$ body weight. SiM $(r=0.92)$ is highly correlated with the gold standard measures of insulin sensitivity in the general population and is an excellent predictor of diabetes, especially in individuals of European extraction [11]. To test the association of statistically inferred haplotypes with continuous traits we used haplotype trend regression [12],

Table 3 Results of individual SNP association analyses

\begin{tabular}{|c|c|c|c|c|c|}
\hline \multirow[t]{2}{*}{ SNP } & \multirow[t]{2}{*}{ Measures } & \multirow{2}{*}{$\begin{array}{l}\text { Number } \\
11 /(12+22)^{a}\end{array}$} & \multicolumn{2}{|c|}{ Mean \pm SD } & \multirow[t]{2}{*}{$p$ from GEE } \\
\hline & & & 11 & $12+22$ & \\
\hline \multirow[t]{9}{*}{ rs4969169 } & Central obesity score ${ }^{b}$ & $2161 / 328$ & $-0.02 \pm 1.01$ & $0.05 \pm 0.87$ & 0.50 \\
\hline & General obesity score ${ }^{b}$ & $2175 / 333$ & $-0.01 \pm 1.00$ & $0.02 \pm 0.89$ & 0.86 \\
\hline & $\mathrm{HOMA}^{\mathrm{b}}$ & $1455 / 224$ & $1.32 \pm 1.03$ & $1.37 \pm 1.29$ & 0.19 \\
\hline & $\mathrm{SiM}^{\mathrm{b}}$ & $593 / 102$ & $91.4 \pm 71.6$ & $79.9 \pm 53.9$ & 0.35 \\
\hline & Triglyceride $^{\mathrm{c}}(\mathrm{mmol} / \mathrm{l})$ & $2027 / 296$ & $1.27 \pm 0.79$ & $1.28 \pm 0.89$ & 0.62 \\
\hline & Total cholesterol ${ }^{\mathrm{c}}(\mathrm{mmol} / \mathrm{l})$ & $2114 / 312$ & $5.57 \pm 1.24$ & $5.58 \pm 1.36$ & 0.63 \\
\hline & HDL cholesterol $^{\mathrm{c}}(\mathrm{mmol} / \mathrm{l})$ & $2122 / 313$ & $1.54 \pm 0.39$ & $1.56 \pm 0.37$ & 0.99 \\
\hline & $\operatorname{ApoA}^{\mathrm{c}}(\mathrm{g} / \mathrm{l})$ & $1982 / 289$ & $1.69 \pm 0.34$ & $1.72 \pm 0.35$ & 0.39 \\
\hline & $\operatorname{ApoB}^{c}(g / 1)$ & $1997 / 289$ & $1.17 \pm 0.36$ & $1.16 \pm 0.36$ & 0.92 \\
\hline \multirow[t]{9}{*}{ rs 12953258} & Central obesity score ${ }^{\mathrm{b}}$ & $2021 / 444$ & $-0.02 \pm 1.00$ & $0.04 \pm 0.95$ & 0.24 \\
\hline & General obesity score ${ }^{b}$ & $2041 / 451$ & $-0.01 \pm 0.99$ & $0.01 \pm 0.97$ & 0.65 \\
\hline & $\mathrm{HOMA}^{\mathrm{b}}$ & $1332 / 323$ & $1.32 \pm 1.03$ & $1.37 \pm 1.26$ & 0.36 \\
\hline & $\mathrm{SiM}^{\mathrm{b}}$ & $555 / 133$ & $91.6 \pm 72.7$ & $84.4 \pm 58.2$ & 0.56 \\
\hline & Triglyceride $^{\mathrm{c}}(\mathrm{mmol} / \mathrm{l})$ & $1873 / 419$ & $1.28 \pm 0.80$ & $1.21 \pm 0.81$ & 0.44 \\
\hline & Total cholesterol ${ }^{\mathrm{c}}(\mathrm{mmol} / \mathrm{l})$ & $1964 / 437$ & $5.59 \pm 1.24$ & $5.49 \pm 1.31$ & 0.24 \\
\hline & $\mathrm{HDL}$ cholesterol $^{\mathrm{c}}(\mathrm{mmol} / \mathrm{l})$ & $1969 / 441$ & $1.55 \pm 0.39$ & $1.55 \pm 0.37$ & 0.75 \\
\hline & $\mathrm{ApoA}^{\mathrm{c}}(\mathrm{g} / \mathrm{l})$ & $1831 / 414$ & $1.70 \pm 0.34$ & $1.70 \pm 0.32$ & 0.78 \\
\hline & $\operatorname{ApoB}^{c}(g / 1)$ & $1848 / 414$ & $1.18 \pm 0.36$ & $1.13 \pm 0.33$ & 0.37 \\
\hline \multirow[t]{9}{*}{ rs8064821 } & Central obesity score ${ }^{b}$ & $2030 / 504$ & $-0.01 \pm 1.00$ & $-0.05 \pm 0.95$ & 0.23 \\
\hline & General obesity score ${ }^{b}$ & $2049 / 508$ & $-0.00 \pm 1.00$ & $-0.04 \pm 0.94$ & 0.23 \\
\hline & $\mathrm{HOMA}^{\mathrm{b}}$ & $1379 / 339$ & $1.29 \pm 0.96$ & $1.43 \pm 1.50$ & 0.26 \\
\hline & $\mathrm{SiM}^{\mathrm{b}}$ & $565 / 137$ & $89.3 \pm 68.6$ & $92.2 \pm 70.0$ & 0.25 \\
\hline & Triglyceride $^{c}(\mathrm{mmol} / \mathrm{l})$ & $1899 / 464$ & $1.27 \pm 0.81$ & $1.25 \pm 0.77$ & 0.39 \\
\hline & Total cholesterol $^{\mathrm{c}}(\mathrm{mmol} / \mathrm{l})$ & $1980 / 490$ & $5.55 \pm 1.24$ & $5.59 \pm 1.27$ & 0.76 \\
\hline & $\mathrm{HDL}$ cholesterol $^{\mathrm{c}}(\mathrm{mmol} / \mathrm{l})$ & $1990 / 491$ & $1.55 \pm 0.39$ & $1.54 \pm 0.38$ & 0.67 \\
\hline & $\operatorname{ApoA1}^{\mathrm{c}}(\mathrm{g} / \mathrm{l})$ & $1853 / 456$ & $1.70 \pm 0.35$ & $1.67 \pm 0.32$ & 0.11 \\
\hline & $\operatorname{ApoB}^{c}(g / l)$ & $1870 / 458$ & $1.17 \pm 0.36$ & $1.18 \pm 0.36$ & 0.78 \\
\hline
\end{tabular}

\footnotetext{
${ }^{\mathrm{a}} 1=$ major allele, $2=$ minor allele; ${ }^{\mathrm{b}}$ age-adjusted $p$ value; ${ }^{\mathrm{c}}$ age- and fasting-adjusted $p$ value. GEE, generalised estimating equations
} 
with the probabilities of haplotype pairs estimated by PHASE 2.0 software [13]. Haplotypes with estimated frequencies below $3 \%$ were pooled together and included in the model as one term. The most frequent haplotype was used as the baseline haplotype with which effects of the other haplotypes were contrasted. To control for population stratification bias, dizygotic (DZ) twin pairs discordant for genotype were also used in a sibling transmission disequilibrium test (sib TDT) association analysis as described elsewhere [14]. Hardy-Weinberg equilibrium was tested by a $\chi^{2}$ test with 1 degree of freedom in one twin of each pair, chosen at random to prevent inflated significance. Analyses of genotype-phenotype association included general tests for single SNPs and haplotypes as well as the sib-TDT for single SNPs. For all the phenotypes, age and menopausal status were included as covariates in the models. For lipids, we additionally included fasting status, BMI and hormone replacement therapy as covariates (the latter affecting $17.5 \%$ of the sample). To reduce the likelihood of identifying false-positive associations, single variables characterising obesity and insulin resistance were analysed only if initial tests with combined variable scores yielded a positive association. Phenotypes significantly $(p<0.05)$ deviating from normal were log-transformed to obtain normal distributions prior to analysis. A $p$ value of $\leq 0.05$ was considered to be statistically significant.

\section{Results}

Only three out of nine SNPs on the SNP databases, rs4969169, rs12953258 and rs8064821, were relatively common, with rare allele frequencies $>6 \%$. Pairwise linkage disequilibrium quantified by $D^{\prime} / r^{2}$ was significant $(p<0.05)$ for all pairwise combinations of the three SNPs: rs12953258 vs rs4969169: 0.845/0.499 $\quad\left(\chi^{2}=1974.5\right.$; $p \leq 0.0001) ; \quad$ rs 8064821 vs $\quad$ rs4969169: $-0.840 / 0.006$ $\left(\chi^{2}=22.73 ; p=1.864 \times 10^{-6}\right)$; rs 8064821 vs $r 12953258$ : $-0.678 / 0.005\left(\chi^{2}=20.57 ; p=5.233 \times 10^{-6}\right)$.

SNP allele frequencies that did not deviate significantly from Hardy-Weinberg equilibrium and haplotype frequencies are given in Table 2. Neither the single SNP analyses (including sib-TDT) nor the haplotype analyses showed any significant associations with obesity or insulin sensitivity measures (see Table 3 for SNP association results). In addition to the lack of any main effects, none of the interactions between age/menopause and SNP/haplotype on the combined obesity and insulin sensitivity scores was significant; therefore, associations with individual obesity and insulin sensitivity variables were not tested.

We found no association of single SNPs and haplotypes with serum lipid variables (Table 3 ). However, a significant interaction between the rs 8064821 and BMI was observed for both triglyceride; $(p=0.022)$ and total cholesterol; $(p=0.007)$. Carriers of the minor allele (allele 2) showed protection against increases in triglyceride and total cholesterol with higher BMI. Haplotype analyses confirmed these interactions. We also observed a significant interaction between rs4969169 and BMI for apolipoprotein
AI (apoAI; $p=0.020$ ), with only allele 1 (major allele) homozygotes showing a significant decrease in apoAI level with the increase in BMI. This effect on apoAI was also confirmed in haplotype analysis. However, the rs8064821/ rs4969169-BMI or haplotype-BMI interactions only accounted for $0.17-0.41 \%$ of the variance in lipid levels.

\section{Discussion}

The current study has two key results. First, we did not find any association between the three SNPs in SOCS3 and obesity, insulin or lipid measures in this study. Second, SOCS3 influences the lipid profile at higher levels of BMI. Our findings in these twin subjects can be considered as representative of the UK female population. We have previously found few differences between twins and singletons in the population generally [6], the only indication being that monozygotic twins had a slightly lower weight and a smaller variance for weight than dizygotic twins and singletons [6]. Others have found that, for example, mortality in twins is no different to that of the general population [15]. Although SOCS3 is an excellent candidate, our results do not support a major role for SOCS3 variants in body weight regulation in our female population. In addition to the lack of any main effects, none of the interactions between age/menopause and SNPs/ haplotype on the combined obesity and insulin sensitivity scores were significant; therefore, associations with individual obesity and insulin sensitivity variables were not tested. The current study had $80 \%(\alpha=0.05)$ power to detect a bi-allelic quantitative trait locus [16], explaining as little as $0.5 \%$ of the variance in HOMA index $(n=1780)$ and $1.1 \%$ of the variance in SiM ( $n=733)$, thereby excluding the possibility that there was a lack of statistical power to detect SNPs with small effects. A second advantage of our study was the availability of comprehensive and accurate measurements of the phenotypes of interest: general and central obesity.

In an effort to repeat the previously reported association between rs 12953258 and insulin sensitivity in 380 healthy young Danish subjects [4] in which rs12953258 showed a recessive effect, we re-analysed the rs 12953258 by using a recessive model but did not observe any significant association with our insulin sensitivity scores. Our main measure of insulin sensitivity (SiM) was based on an oral rather than an intravenously administered glucose load, but this is unlikely to be the major reason for the different findings. More likely explanations include different environmental exposures, different patterns of linkage disequilibrium or different sampling variation among the populations.

Exploratory analyses showing interaction of lipid levels with BMI were not part of the a priori hypothesis and should be viewed with caution. An influence of SOCS-3 on circulating lipids remains possible through activation of sterol regulatory binding element binding protein-1c and subsequent stimulation of hepatic fatty acid synthesis [17]. However, a significant interaction between rs8064821 and 
BMI for serum triglyceride and total cholesterol was demonstrated by a diminished increase in triglyceride and total cholesterol with higher BMI in carriers of the rare allele. The inclusion of haplotype-BMI interactions improved the overall model for total cholesterol and triglyceride because of the protective effects of haplotype 112. A significant interaction between rs4969169 and BMI was found for apoAI; a reduction in apoAI with higher BMI occurred in common allele homozygotes, which was confirmed in haplotype analysis. Neither SNP, however, appears to have functional potential. In contrast, the rare A allele of the third SNP that we tested without evidence of main effect or interaction, rs 12953258 , deletes an activator protein 2 transcription factor binding site (AP2) [4], which among other effects, is involved in chronic inflammation. The common C allele destroys a site bound by ZNF202, a transcriptional repressor binding to elements found predominantly in genes involved in lipid metabolism (TRANSFAC database, available from http://www.generegulation.com/cgi-bin/pub/databases/transfac/search.cgi, last accessed in November 2005). This variant of SOCS3 may yet prove influential in determining a subject's lipid profile.

In summary, our results suggest that polymorphisms in SOCS3 are not associated with obesity or insulin resistance measurements. However, this preliminary study does not exclude a strong genetic determination of SOCS-3 variability. As yet, no conclusion is possible regarding whether variable SOCS3 expression or function is involved in insulin or lipid metabolism.

Acknowledgement This study was funded by the Wellcome Trust (project grant no. 073142). The Twin Research and Genetic Epidemiology Unit received support from the Wellcome Trust, Arthritis Research Campaign, the Chronic Disease Research Foundation and the European Union 5th Framework Programme GenomEUtwin (EU contract no. QLG2-CT-2002-01254). This research was conducted within the network of the London Innovation, Dissemination, Evaluation and Application Strategy (IDEAS) Genetic Knowledge Park and utilised the St George's, University of London Medical Biomics Centre.

\section{References}

1. Krebs DL, Hilton DJ (2001) SOCS proteins: negative regulators of cytokine signaling. Stem Cells 19:378-387

2. Munzberg H, Myers MG (2005) Molecular and anatomical determinants of central leptin resistance. Nat Neurosci 8:566570
3. Pirola L, Johnston AM, Van Obberghen E (2004) Modulation of insulin action. Diabetologia 47:170-184

4. Gylvin T, Nolsoe R, Hansen T et al (2004) Mutation analysis of suppressor of cytokine signalling 3 , a candidate gene in Type 1 diabetes and insulin sensitivity. Diabetologia 47:1273-1277

5. Spector TD, MacGregor AJ (2002) The St. Thomas' UK Adult Twin Registry. Twin Res 5:440-443

6. Andrew T, Hart DJ, Snieder H, de Lange M, Spector TD, MacGregor AJ. (2001) Are twins and singletons comparable? A study of disease-related and lifestyle characteristics in adult women. Twin Res 4:464-477

7. Middelberg RP, Spector TD, Swaminathan R, Snieder H (2002) Genetic and environmental influences on lipids, lipoproteins, and apolipoproteins: effects of menopause. Arterioscler Thromb Vasc Biol 22:1142-1147

8. de Lange M, Snieder H, Ariens RA, Andrew T, Grant PJ, Spector TD (2003) The relation between insulin resistance and hemostasis: pleiotropic genes and common environment. Twin Res 6:152-161

9. Trégouët D-A, Ducimetère P, Tiret, L (1997) Testing association between candidate-gene markers and phenotype in related individuals, by use of estimating equations. Am J Hum Genet 61:189-199

10. Avignon A, Boegner C, Mariano-Goulart D, Colette C, Monnier L (1999) Assessment of insulin sensitivity from plasma insulin and glucose in the fasting or post oral glucoseload state. Int J Obes Relat Metab Disord 23:512-517

11. Hanley AJ, Williams K, Gonzalez C, D'Agostino RB, Jr, Wagenknecht LE, Stern MP, Haffner SM (2003) Prediction of type 2 diabetes using simple measures of insulin resistance: combined results from the San Antonio Heart Study, the Mexico City Diabetes Study, and the Insulin Resistance Atherosclerosis Study. Diabetes 52:463-469

12. Zaykin DV, Westfall PH, Young SS, Karnoub MA, Wagner MJ, Ehm MG (2002) Testing association of statistically inferred haplotypes with discrete and continuous traits in samples of unrelated individuals. Hum Hered 53:79-91

13. Stephens M, Donnelly P (2003) A comparison of bayesian methods for haplotype reconstruction from population genotype data. Am J Hum Genet 73:1162-1169

14. Dong Y, Zhu H, Wang X, Dalageorgou C, Carter N, Spector TD, Snieder H (2004) Obesity reveals an association between blood pressure and the G-protein beta3-subunit gene: a study of female dizygotic twins. Pharmacogenetics 14:419-427

15. Christensen K, Vaupel J, Holm NV, Yashin AI (1995) Twin mortality after age six: fetal origin hypothesis versus twin method. BMJ 310:432-436

16. Sham PC, Cherny SS, Purcell S, Hewitt JK (2000) Power of linkage versus association analysis of quantitative traits, by use of variance-components models, for sibship data. Am J Hum Genet 66:1616-1630

17. Ueki K, Kondo T, Tseng YH, Kahn CR (2004) Central role of suppressors of cytokine signaling proteins in hepatic steatosis, insulin resistance, and the metabolic syndrome in the mouse. Proc Natl Acad Sci USA 101:10422-10427 